\title{
Mental Health First Aid (MHFA): A First-Hand Look at Why You Should Consider this Training for Your Campus Community
}

\author{
Author \\ David J. Denino, LPC, NCC \\ American Red Cross/QPR Institute
}

\begin{abstract}
Mental Health First Aid, an eight-hour course that gives people the skills to help someone who is developing a mental health problem or experiencing a mental health crisis, can give your campus yet another tool to help in the identification of students, faculty, and staff who may be experiencing mental health issues so that you can intervene before those issues reach the crisis stage.
\end{abstract}




\section{Introduction}

Hello ALGEE... The five basic tenets of Mental Health First Aid (MHFA) are represented by this acronym, and there's more about ALGEE later. What you are about to read are my personal experiences and recommendation that you consider for implementation of MHFA at your campus. A five-day training offered by MHFA has reinforced my extensive background in both professional and volunteer training, and I recently became a certified instructor for the standard/adult MHFA curriculum.

In this article, I will offer my personal experiences with this program to help the reader better understand MHFA. Prior to this training, my work in the arena of crisis response, suicide prevention/postvention, disaster mental health, and psychological first aid spanned four decades. My training has included serving in leadership roles at a mid-size state university, as an adjunct professor in the area of clinical mental health, as a mental health lead for American Red Cross Disaster Services (in Conn. and RI), as a Master Trainer for QPR, and as an instructor for Psychological First Aid/Fundamentals of Disaster Mental Health. My crisis intervention work has also been regional and national, working at such events as Superstorm Sandy, Hurricanes Katrina and Irene, and the school shootings at Sandy Hook, Conn. Having participated in multiple types of trainings that would benefit campus communities, I present a synopsis of MHFA training for your consideration. By no means is it a product endorsement, as I have always affiliated myself with multiple programs that enhance outreach and support the well being of students and staff. MHFA may become another item in your toolkit by which to measure student health, safety, and the effectiveness of prevention programing.

First, allow me to provide a brief review of what MHFA is for those of you who are new to it, along with a brief update for those who may already be acquainted with the program.

\section{What is Mental Health First Aid?}

Mental Health First Aid is an eight-hour course that gives people the skills to help someone who is developing a mental health problem or experiencing a mental health crisis. The evidence behind the program demonstrates that it does build mental health literacy, helping the public identify, understand, and respond to signs of mental illness.

The National Council for Behavioral Health defines it this way: "Mental Health First Aid is an in-person training that teaches you how to help people developing a mental illness or in a crisis." Mental Health First Aid USA is managed, operated, and disseminated by the National Council for Behavioral Health, Maryland Department of Health and Mental Hygiene, and the Missouri Department of Mental Health.
It is important to note that Mental Health First Aid USA is listed in the Substance Abuse and Mental Health Services Administration's National Registry of Evidence-Based Programs and Practices (other programs like QPR for suicide prevention are also listed). Basing campus programming in proven approaches is essential.

A personal note about programs like MHFA: As a Connecticut resident, I was privileged to have our state senator, Richard Blumenthal (D-CT) sponsor The Mental Health First Aid Act (S. 711/H.R. 1877) in the U.S. Senate, along with Senator Kelly Ayotte (R-NH). Although the act is not a direct endorsement of any one program, the Australian-based MHFA does fit the "bill."

\section{General Review of Training:}

MHFA training provides an intensive immersion into mental health issues across the spectrum. It is a five-day experience that is made highly palatable by very knowledgeable instructors. Two certifications can be earned at the end of training. Completion of an eight-hour course certifies/trains you to provide initial help to people experiencing mental health problems such as depression, anxiety disorders, psychosis, and substance abuse disorders, and instructor certification qualifies you to actually teach the aforementioned eight-hour curriculum.

Make no mistake; it's a long week, and for appropriate reasons. The eight-hour course is conducted during the first two-day training. The remaining three days are devoted to having participants actually teach sections of the curriculum. MHFA instructors do an excellent job of helping all contributors build their comfort level with the material. There are well-experienced teachers, professors, and trainers alongside the first-timers. This coalesces to be manageable for each person through the teamwork, comfort, and leadership MHFA instructors provide. Essential to the process is the immediate feedback offered by the MHFA instructors for each section taught. The final step has instructors meet individually with attendees for one-to-one feedback.

\section{Depth of Curriculum}

First two days: MHFA uses the term "unpack" for all the parts for this two-day experience. It's a reasonable description, as it is sort of like you have gone on a working vacation and they brought the suitcase. The entire curriculum is arranged around two booklets that are unpacked in parts. Both days are broken into sessions using the booklets provided. One booklet is what all participates/trainers use for the eight-hour training, complimented by a trainers' teaching guide. The MHFA instructors present all materials supplemented by PowerPoint slides. All of the work is interactive, and has participants working in large and small group formats. Plenty of time is provided to model the "how to" functions of the content, and instructors are very supportive in coaching participants. 
The seminal points made the first two days are:

- What MHFA is and the role of the Mental Health First Aider.

- The prevalence and impact of mental health issues in the United States.

- Introduction of the MHFA Action Plan.

- An overview of the signs, symptoms, and possible risk factors/warning signs of depression and anxiety.

Once the eight-hour training is completed over the first two days, you move on to becoming certified to teach over the next three days.

Next three days: The next suitcase to unpack is indeed the largest one. The entire curriculum from the prior days is divided up into various components. All participants are assigned section(s) to present in 50-minute sessions. MHFA provides you with the topic, exercises and discussion points, corresponding slides to the PowerPoint, and the reference pages to the teaching guide. An example of the exercise and discussion would be:

- Topic: Depression and Anxiety/Types of Mood Disorders/ Signs \& Symptoms of Depression.

- Exercises and Discussion: Signs \& Symptoms of Depression and Anxiety; DVD: The Pain of Depression.

- Slides 12-16 for presentation and pages 16-19 for teaching notes.

Throughout the training, a great deal of emphasis is placed on coaching and feedback, as the MHFA instructors and fellow participants support each student-presenter. These days are devoted to truly dissecting the entire eight-hour curriculum, with a strong emphasis on preserving its integrity, efficacy, and fidelity. You learn about:

- Depression and mood disorders.

- Anxiety disorders.

- Trauma.

- Psychosis.

- Substance use disorders.

Mental Health First Aid teaches about recovery and resiliency — "the belief that individuals experiencing these challenges can and do get better, and use their strengths to stay well," notes the National Council for Behavioral Health.

\section{Understanding ALGEE}

The whole training is to support ALGEE, a five-step action plan designed to help individuals assess a situation and help. It calls for:

1. Assessing for risk of suicide or harm.
2. Listening nonjudgmentally.

3. Giving reassurance and information.

4. Encouraging appropriate professional help.

5. Encouraging self-help and other support strategies.

\section{Potential for Campus Use}

Mental health issues on college and university campuses mirror what any community might experience. Our advantage is that we have neighborhoods built in that include students, faculty, student affairs staff, police, facilities operations, clerical staff, and a myriad of others who can easily become the "eyes and ears" on the ground to ensure that those who might be experiencing mental health issues are identified and helped.

Should you spend the money to have someone on staff trained to teach MHFA on your campus? Budgets were always the bane of my existence, as they are for many campus leaders across the country. Cut, cut more, and cut even that. We all became more lean and resourceful in our financial stewardship, and ask how can we could maximize every last dollar? When it comes to whether to spend money to train someone in MHFA, my simple answer is yes. One or two staff members who are trained to teach this is a good expenditure that will offer an invaluable return. Up-front costs are mitigated by the number of times that you can offer MHFA on your campus. One person can be trained to offer MHFA for approximately $\$ 2,000$, and costs for materials per person are very low. Additionally, there are opportunities to award continuing education units. (Check the websites below for more information.)

By way of the Mental Health First Aid Act, our nation has recognized the need for programs that will provide benefits across many different kinds of settings. Three basic doctrines they outlined in that legislation mentioned earlier were:

- Recognizing the symptoms of common mental illnesses and substance use disorders.

- De-escalating crisis situations safely.

- Initiating timely referral to mental health and substance abuse resources available.

These are three things that we all endeavor to do well on our campuses, and MHFA can help enhance the good work we all do.

\section{Helpful Web Resources:}

Mental Health First Aid USA: www. mentalhealthfirstaid.org. Frequently Asked Questions: www.mentalhealthfirstaid.org/ cs/faq/. 